cancer risk in epidemiological and/or animal studies. Consistent with our finding, shorter RTL with 2,4-D use was previously observed in an analysis of buccal cells in the AHS.

\section{WORK RELATED MORTALITY AND HOSPITAL ADMISSIONS AMONG MIGRANT WORKERS IN AUSTRALIA, 1991-2010}

${ }^{1}$ Alison Reid, ${ }^{1}$ Susan Peters, ${ }^{1}$ Nieves Felipe, ${ }^{2}$ Erik Lenguerrand, ${ }^{2}$ Seeromanie Harding. ${ }^{1}$ Harry Perkins Institute of Medical Research, The University of Western Australia, Perth, Western Australia, Australia; ${ }^{2}$ Social and Public Health Sciences Unit, University of Glasgow, Glasgow, UK

\subsection{6/oemed-2014-102362.46}

Objectives One in four of the Australian population is born abroad, with skilled migration encouraged since the mid-1990s. The shift from an industrial to a service based economy has seen a decline in recent decades of work-related injuries (WRIs) and related mortality in Australia. We examine deaths and hospital admissions from WRI, among foreign and Australian-born workers.

Method Work-related mortality and hospital admission rates were derived from tabulated data from the 1991 to 2011 censuses, 1991-2002 national deaths and hospital admissions for 2001-2010. Comparisons across country of birth groups were conducted using incidence rate ratios (IRRs). Gender specific mortality and hospital admission rates were directly standardised (DSRs) using the World Standard Population. Negative binomial regression models compared the country-specific mortality and hospital admission rates of the foreign-born workers with those of Australian-born workers.

Results DSRs and IRRs were generally higher for Australianborn than foreign-born workers. A notable exception was New Zealand born men, among whom there was a $10 \%(95 \% \mathrm{CI}$ 9.1-13.1) excess mortality and 24\% (95\% CI 12.2-12.6) excess hospital admissions. Adjusting for age, gender, year and skill level removed the differences in risk of WRI death between Australian and foreign-born workers.

Conclusions These findings show a reversal of the historic trend of foreign-born workers being at higher risk than the local-born. They signal a need to promote healthy work environments in all industries to fur.ther reduce the risk of WRI to all workers in Australia.

\section{TAKING RISKS AND SURVIVAL JOBS: FOREIGN-BORN WORKERS AND WORK-RELATED INJURIES IN AUSTRALIA}

${ }^{1}$ Alison Reid, ${ }^{2}$ Erik Lenguerrand, 'Iracema Santos, ${ }^{2}$ Ursula Read, ${ }^{3}$ Anthony LaMontagne, ${ }^{1}$ Lin Fritschi, ${ }^{2}$ Seeromanie Harding. ${ }^{1}$ Harry Perkins Institute of Medical Research, The University of Western Australia, Perth, Western Australia, Australia; ${ }^{2}$ Social and Public Health Sciences Unit, University of Glasgow, Glasgow, UK; ${ }^{3}$ VicHealth Centre for the Promotion of Mental Health and Community Wellbeing, Melbourne School of Population Health, University of Melbourne, Melbourne, Victoria, Australia

\subsection{6/oemed-2014-102362.47}

Objectives Higher rates of work-related injuries (WRI) have been reported among foreign-born workers in many countries, but little is known about the situation in Australia. The aim of the study was to examine WRI among foreign-born workers in Australia.
Method This was a two phase mixed methods study. The first stage used the 2005/6 and 2009/10 Australian national MultiPurpose Household Survey (MPHS) information on WRI occurring in the previous year $(\mathrm{N}=36702)$. Logistic regression examined the relationship between WRI and region of birth (Australia born =baseline), and whether the effect varied by period of arrival in Australia, age, sex, industry and working conditions. In the second stage, purposively sampled foreignborn workers and stakeholders from 22 countries took part in individual interviews $(n=17)$ or focus groups $(n=75)$. A concurrent thematic analysis was conducted.

Results In the MPHS, more WRI were reported by workers in agriculture, manufacturing, construction, hospitality and transport industries than in the service industry, and by those in unfavourable working conditions (e.g. unpaid leave). Migrant status, regardless of region of birth, was generally not associated with higher WRI reporting. Qualitative interviews suggested that understanding and practice of Occupational Health and Safety (OH\&S) was influenced by education, skill level, job security, dependent relatives in home countries and by social ties in communities.

Conclusions Whereas the MPHS point to higher risks related to area of work rather than migrant status, qualitative interviews suggest under-reporting of WRI among low income migrants mainly due to fear of losing their jobs.

\section{INCREASED RISK OF SPONTANEOUS ABORTION AND MENSTRUAL ABERRATIONS IN FEMALE WORKERS IN SEMICONDUCTOR INDUSTRY, SOUTH KOREA}

${ }^{1}$ Inah Kim, ${ }^{2}$ Myoung-Hee Kim, ${ }^{3}$ Sinye Lim. ${ }^{1}$ Graduate School of Public Health, Yonsei University, Seoul, Republic of Korea; ${ }^{2}$ People's Health Institute, Seoul, Republic of Korea; ${ }^{3}$ Kyung Hee University, Seoul, Republic of Korea

\subsection{6/oemed-2014-102362.48}

Objectives Since a series of papers to report reproductive problems among semiconductor workers in mid-2000s, there have been few studies to monitor such issues in spite of global expansion and technical development of semiconductor industry. In such a context, we examined reproductive risk among female workers in Korea.

Method Based on the claim data of the National Health Insurance from 2008 to 2012, we estimated age-specific rates of spontaneous abortion (SA) and menstrual aberration (MA) among women aged 20 to 39 years, and then compared them between exposure and control groups using relative risk (RR). Semiconductor workers were defined as exposure group, and three different groups were used as controls; first, economically inactive women, second, working population in total, and last, workers employed in bank industry.

Results Female workers in semiconductor industry have significantly higher risk for SA (RR 1.57 and 1.58 for 20-29 and 3039 years old, respectively) and MA (RR 1.54 and 1.25 for 20 29 and 30-39 years old, respectively) compared to economically inactive population. RRs for SA (RR 1.40 and 1.67) and MA (RR 1.38 and 1.35) were significantly higher compared to working population in total. RRs for SA and MA also were significantly higher compared to workers employed in bank except for RR of SA for the twenties.

Conclusions In spite of technical innovations and health and safety measures taken by semiconductor industry, workers seem to be still exposed to reproductive risk, at least in Korea. Further studies to identify specific hazards should be launched. 